\title{
Análise da variabilidade na produção de pães em uma pequena padaria com o auxílio do controle estatístico da qualidade
}

\author{
RESUMO
}

Edvan Araújo de Sousa Júnior edvanjrx@hotmail.com

Federal de Minas Gerais (IFMG), Congonhas, Minas Gerais, Brasil

\section{Tatielle Menolli Longhini} tatielle.longhini@gmail.com Universidade Federal de Minas Gerais (IFMG), Belo Horizonte, Minas Gerais, Brasil

Hanah Ingred da Silva Costa hanahcmd@hotmail.com Fundação Getúlio Vargas (FGV), Belo Horizonte, Minas Gerais, Brasil
Em processos produtivos a padronização de qualidade é imprescindível, tanto para maior eficácia e eficiência da organização, quanto para satisfação dos clientes. O presente artigo objetiva aplicar ferramentas de qualidade em uma pequena padaria no município de Congonhas - MG. Em específico, busca identificar a variabilidade de peso na fabricação do pão francês e as possíveis causas especiais presentes no processo. Mesmo com a adoção do padrão do Inmetro, tal estudo se propõe a definir qual a melhor forma de comercialização de pães: por unidade ou por peso. Para isso, fez-se o estudo de caso através de métodos de controle estatístico da qualidade, onde a realidade foi avaliada por diagrama de Ishikawa, cartas de controle, curva de poder e capacidade do processo. Como resultado, os mecanismos de controle estatístico do processo indicaram um descontrole no procedimento de fabricação, o que aponta a necessidade de venda por peso.

PALAVRAS-CHAVE: Controle estatístico da qualidade. Ferramentas da qualidade. Variabilidade. Causas especiais. Inmetro. 


\section{INTRODUÇÃO}

A preocupação em torno da qualidade de produtos e serviços é própria das organizações. Qualidade influi em custos de produção e atendimento de requisitos de mercado, o que determina diretamente sua atuação, desde seu desenvolvimento até a comercialização. Para que seja garantida, busca-se ajustar as interferências internas e externas que, em grande maioria, impactam negativamente no processo na obtenção de um produto/serviço final (PALADINI, 2002; CARVALHO, PALADINI, 2005).

Em decorrência disso, é comum o uso de ferramentas de Controle Estatístico da Qualidade (CEQ) para o monitoramento da variabilidade do processo produtivo. A variabilidade pode ser descrita principalmente por métodos estatísticos, no qual desempenham um papel central em toda engenharia da qualidade (MONTGOMERY, 2009). O Controle Estatístico do Processo (CEP) é usado para estabelecer um padrão de qualidade aceitável.

Segundo Ramos (2000), o controle estatístico do processo é uma das mais poderosas metodologias desenvolvidas visando o controle eficaz da qualidade. Entretanto, partindo do aspecto empresarial do Brasil, que é composto em sua maioria por micro e pequenas empresas, ainda é notável o baixo uso da ciência estatística em prol da sua atividade-fim (RAMOS, 2000).

O uso da metodologia estatística não assegura a solução de todas as problemáticas de um processo produtivo. Mas fornece um formato racional e lógico para determinação das causas e desenvolvimento de soluções. Assim, podese alcançar a melhoria contínua nos sistemas, incorrendo em maior produtividade (CARNEIRO NETO, 2003; MOREIRA, 2004).

O CEP é composto por ferramentas de monitoramento detalhado de comportamento dos processos, onde é possível detectar as causas de variabilidade e instabilidade (CORTIVO, 2005). Abrange, portanto, os estágios de coleta, avaliação e interpretação de dados para a tomada de decisão e solução do problema em particular (PARANTHAMAN, 1990). É comum, para isso, fazer uso de ferramentas tais como o Diagrama de Ishikawa, as cartas de controle, além da análise da estabilidade, poder e capacidade do processo (COSTA, EPPRECHT, CARPINETTI, 2005).

No Brasil, o setor de panificação é diversificado e representativo. Estima-se a operação de aproximadamente $63 \mathrm{mil}$ estabelecimentos, sendo $96 \%$ deles composto por micro e pequenas empresas (SEBRAE, 2010). O comércio de pão francês, em específico, passou a ser discutido e regularizado pelo Inmetro em 1994. Isso porque, a variabilidade de fabricação era representativa, e antes a comercialização se dava por unidade do produto, e não por peso.

Desse modo, o objetivo deste artigo é aplicar em uma microempresa panificadora metodologias de CEQ em seu processo, de maneira que seja avaliada sua capacidade produtiva e a variabilidade de fabricação. A partir de tal avaliação, pode-se auxiliar a tomada de decisão dos gestores quanto à venda de pães por unidades ou por quilo. 


\section{REFERENCIAL TEÓRICO}

\section{CONTEXTO DA ATIVIDADE DE PANIFICAÇÃO: NORMAS E PADRONIZAÇÃO}

No Brasil, cerca de 63 mil panificadoras atendem diariamente o número aproximado de 44 milhões de brasileiros. Segundo a Associação Brasileira da Indústria da Panificação e Confeitaria (ABIP), noventa e seis porcento dos estabelecimentos é composto de micro e pequenas empresas (SEBRAE, 2010).

O setor de panificação é bem diversificado, tendo em vista a grande gama de produtos produzidos. Entretanto, é preciso preocupar-se com o processo de fabricação, de tal modo que os produtos sejam de boa qualidade e tenham um padrão uniforme.

De acordo com a ABIP, o pão francês é o produto mais vendido na maioria das padarias brasileiras. Isso mesmo com a mudança de atuação nos últimos anos, em que se tornou comum a venda de produtos de supermercado, de conveniência e bebidas em geral (SEBRAE, 2010). Devido ao destaque, o pão francês foi alvo de padronização pelo Instituto Nacional de Metrologia, Normatização e Qualidade Industrial (Inmetro) por meio da portaria no 017, de 25 de janeiro de 1994. Esta resolve que:

Art. 1ㅇ A fabricação e venda de pão francês ou pão de sal deve ser feita nos seguintes valores para os pesos nominais: $50 \mathrm{~g}$, $100 \mathrm{~g}, 200 \mathrm{~g}, 300 \mathrm{~g}, 500 \mathrm{~g}, 1 \mathrm{Kg}$. Parágrafo primeiro: O pão francês ou pão de sal pode ser comercializado em quaisquer valores inferiores a $30 \mathrm{~g}$. Parágrafo segundo: O produto fabricado segundo o disposto no parágrafo primeiro do presente artigo, só pode ser comercializado por pesagem direta, na presença do consumidor. Art. 2ㅇ A tolerância para o peso do pão francês ou pão de sal será de $5 \%$ (cinco por cento), para mais ou para menos, na média correspondente à amostra retirada conforme o contido no parágrafo único do Artigo 8 o da Portaria Inmetro no 02/82 (INMETRO, 1994, p. 1).

Em 1997, a produção das panificadoras teve adequações pela Portaria no 003, de 10 de janeiro, o que permitiu a venda tanto por unidade ou peso. A padaria que optasse somente pela venda do pão francês pelo peso deveria notificar os consumidores por meio de cartazes de fácil visibilidade (INMETRO, 1997).

Mesmo com o padrão pré-estabelecido, o Inmetro constatou que os pães possuíam uma alta variabilidade, percebida a olho nu. Muitos consumidores questionavam tanto a venda por unidade, quanto por peso. Em decorrência das dúvidas levantadas, o Inmetro lançou a Portaria no 63, de 15 de março de 2006, que consistiu em uma consulta pública sobre comércio do produto somente pelo peso (INMETRO, 2006a).

Consequentemente, o Inmetro elaborou a Portaria no 146, de 20 de junho de 2006, especificando a venda somente por peso. Tal mudança teve o apoio dos sindicatos e associações do setor de panificação. Entrou em vigor 120 dias após sua publicação, tendo em vista o período de adequação por parte dos panificadores de todo país (INMETRO, 2006b). 


\section{CONTROLE ESTATÍSTICO DO PROCESSO (CEP)}

Para Ramos (2000), todo trabalho executado em uma empresa pode ser encarado como um processo. Ou seja, um conjunto de atividades realizadas com um determinado propósito. Em todas as organizações há uma infinidade deles, que são compostos por cinco componentes básicos: fornecedores, entradas, transformação, saídas e clientes.

No entanto, tais processos sempre são suscetíveis a variação do seu valor alvo (ou valor especificado), que nada mais é que as diferenças existentes entre as unidades produzidas (MONTGOMERY, 2009). O objetivo do CEP é incorporar melhorias no sistema produtivo, de modo que as variabilidades sejam controladas e monitoradas (PALADINI, 2002; CARVALHO, PALADINI, 2005).

A variabilidade é justificada por causas aleatórias ou especiais. Todos os processos são vulneráveis a uma variabilidade natural, em decorrência de causas aleatórias. Por outro lado, também são propensos às chamadas causas especiais, que são controláveis, e influencia o deslocamento da média do valor alvo, com aumento da dispersão entre os itens (COSTA, EPPRECHT, CARPINETTI, 2005).

Segundo Costa, Epprecht e Carpinetti (2005) quando o processo está sob causas aleatórias, diz-se que ele está no estado de controle estatístico, ou simplesmente, em controle. Porém, quando o sistema também está sob causas especiais, diz-se que o processo está fora de controle. A Figura 1 (à esquerda) elucida um processo isento a causas especiais, ou seja, um processo apenas com causas aleatórias. Já à direita apresenta um com causas especiais, onde é possível perceber a alteração no valor alvo.

Figura 1 - Processo sob causas aleatórias e especiais

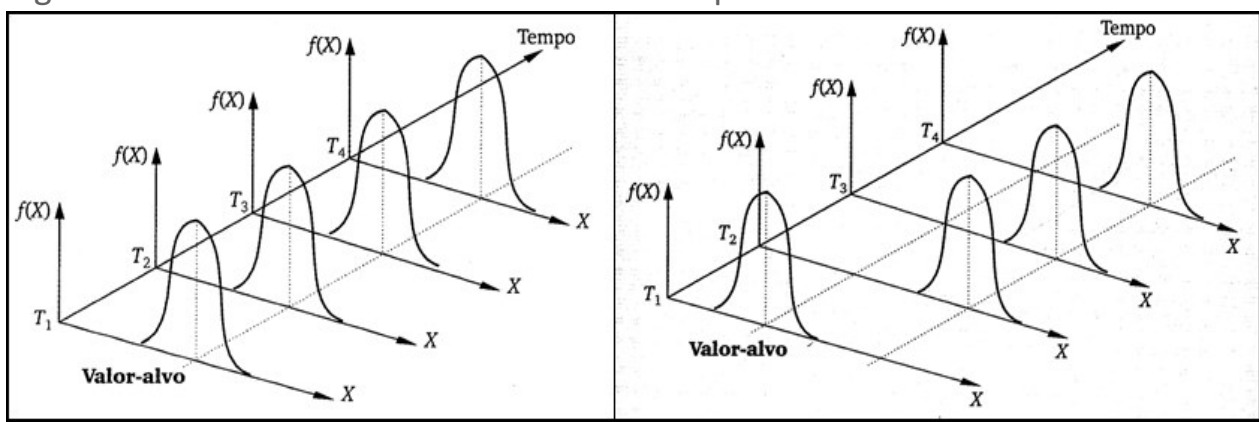

Fonte: Adaptado de Costa, Epprecht e Carpinetti (2005)

A palavra controle pode ter dois significados distintos: fiscalização ou ajuda. Por fiscalização, entende-se o ato de acompanhar para evitar um comportamento indesejado. Já no significado de ajuda, quer-se dizer auxiliar em alguma coisa a obter um melhor desempenho, como é o caso do CEP, conforme descrevem Ramos (2000) e Montgomery (2009).

O CEP prega o controle da qualidade simultâneo com a produção (controle do processo), ao invés da inspeção após a produção, em que se separa os produtos defeituosos dos bons (controle do produto). Logo, o enfoque do controle do processo é a prevenção de defeitos ou erros durante a produção, garantindo uma melhor qualidade, ajuste e controle (RAMOS, 2000). O Quadro 1 compara o controle do produto com o controle do processo, enfatizando o objetivo, o padrão de comparação, e o tipo e responsável pelas ações de controle. 
Quadro 1 - Controle do produto versus controle do processo

\begin{tabular}{|ccc|}
\hline Tipo de Controle & Produto & Processo \\
\hline Ênfase & Detecção de defeitos & Prevenção de defeitos \\
Objetivo & Separar itens bons de ruins & Evitar itens ruins \\
Padrão de Comparação & Limites de Especificação & Limites de Controle \\
Tipo de Ação & Inspeção & Controle \\
Responsável & Operador ou inspetor & Todos os envolvidos \\
\hline
\end{tabular}

Fonte: Adaptado de Ramos (2000).

A metodologia do CEP, portanto, é aplicada de maneira preventiva, utilizandose de análise objetiva. Sua atuação é abrangente, podendo ser aplicado no processo como um todo para avaliação de qualidade e atendimento de requisitos de diferentes áres e setores em análise (PALADINI, 1990; DINIZ, 2001).

No trabalho de Christino, Bonduelle e Iwaraki (2010) buscou-se analisar, à partir de cartas de controle, o comportamento das variáveis que influenciam a qualidade produtiva do setor de moldureira. Os resultados apontaram que todas as variáveis encontravam-se fora de controle estatístico. Já Lima et al. (2006) utilizaram o CEP para avaliação da produção farmacêutica, sendo indicada a não padronização e não conformidade dos processos, o que é indesejado nesse tipo de segmento.

Silva et al. (2007) aplicaram a metodologia do controle estatístico para avaliar a qualidade da colheita, sendo identificadas perdas altas na colheita de algodão, uma vez que a colhedora não possuía boa eficiência. Lindo e Nunes (2011) buscaram analisar os aspectos físicos e químicos na produção de alimentos, monitorando o resultado de nitrogênio total e proteína bruta do peito de frango, conseguindo desenvolver planejamentos para melhoria contínua do processo.

Por fim, Noronha et al. (2011) detectaram o controle da variabilidade de perdas e danos à soqueira, que deve operar dentro de padrões aceitáveis. Após a análise, em diferentes períodos de colheita, notou-se que não há diferença quanto a danos às soqueiras a cada condição analisada, e as perdas ficaram em limites aceitáveis.

O uso da metodologia, portanto, traduz-se em resultados práticos e ajustes importantes para o controle de qualidade, e aumento de eficácia e eficência de produção. Para isso, convém compreender a composição do método, e como proceder as análises para interpretação de resultados e implementação de melhorias.

\section{LIMITES DE CONTROLES E OS LIMITES DE ESPECIFICAÇÃO}

No que se refere aos limites de especificação, Costa, Epprecht e Carpinetti (2005) descrevem que estes são especificados pela engenharia, visando normas ou padrões, aplicando-se a valores individuais. Quando o processo é estável e ajustado, o ideal é que toda a distribuição dos valores esteja dentro dos limites de especificação.

Em função disso, é comum o uso de carta de controles, onde são expostos os limites de controle e a linha central, como forma de identificar ocorrências que extrapolam os limites. $\mathrm{O}$ que pode apontar a presença de causas especiais que venham a explicar a variabilidade do processo (JURAN, 1992; MONTGOMERY, 
2009). Ou seja, caso os pontos ocorrerem além dos limites de controle, julga-se o processo como fora de controle (VIEIRA, 1999).

É importante verificar se o processo atende às especificações, o que se faz com o estudo de capacidade (RAMOS, 2003). Com ele, analisa-se se o processo mantém as tolerâncias e garante embasamento para a tomada de decisão da gestão (MONTGOMERY, 2009; SANTOS, BATISTA, 2005).

Para tanto, os Índices de Capacidade de Processo (ICP's) são parâmetros adimensionais que indiretamente medem o quanto o processo consegue atender às especificações, assim sendo, quando maior os índices, melhor o processo estará atendendo às especificações. Usualmente, os mais conhecidos são: $\mathrm{Cp}, \mathrm{Cpk}$ e o $\mathrm{Cpm}$, sendo que para o Cpk admite-se a classificação: processo incapaz $(\mathrm{Cpk}<1)$, razoavelmente capaz $(1 \leq \mathrm{Cpk} \geq 1,33)$ e processo capaz $(\mathrm{Cpk}>1,33)$. Matematicamente, são definidos pela Equações 1, 2 e 3.

$$
\begin{aligned}
& C p=\frac{L S E-L I E}{6 \sigma} \\
& C p k=\operatorname{Min}\left\{\frac{L S E-\mu}{3 \sigma} \mid \frac{\mu-L I E}{3 \sigma}\right\} \\
& C p m=\frac{L S E-L I E}{6 \sqrt{\sigma^{2}+(d-\mu)^{2}}}
\end{aligned}
$$

Onde:

LSE e LIE: Limite Superior de Especificação e Limite Inferior de Especificação, respectivamente;

$\mu$ : média dos itens;

б: desvio padrão dos itens;

d: ponto médio do intervalo de especificação.

Logo, o processo de controle deve ser constantemente monitorado, para detectar a presença de causas especiais. Assim, deve-se proceder a investigação para identificar as causas especiais e intervir para eliminá-las. Sendo assim, monitorar e identificar, respectivamente, é possível por meio de dois métodos: os gráficos de controle e o diagrama de causa e efeito (diagrama de Ishikawa).

Os gráficos de controle de (valor alvo, ou a média das amostras) e R (amplitude das amostras), também conhecidos respectivamente por gráfico da média e gráfico da amplitude, servem para monitorar processos cuja característica de qualidade de interesse é uma grandeza mensurável (THOMPSON, KORONACKI, 1993). O monitoramento é realizado através da análise das amostras: a cada intervalo de tempo retira-se uma amostra de $\mathrm{n}$ itens para a análise. Para cada amostra, é calculada a média dos valores medidos e a amplitude amostral $\mathrm{R}$ e ao final são plotados nos gráficos, conforme expõe e ilustra a Figura 2 de Costa, Epprecht e Carpinetti (2005). 
Figura 2 - Gráficos de controle de X e R

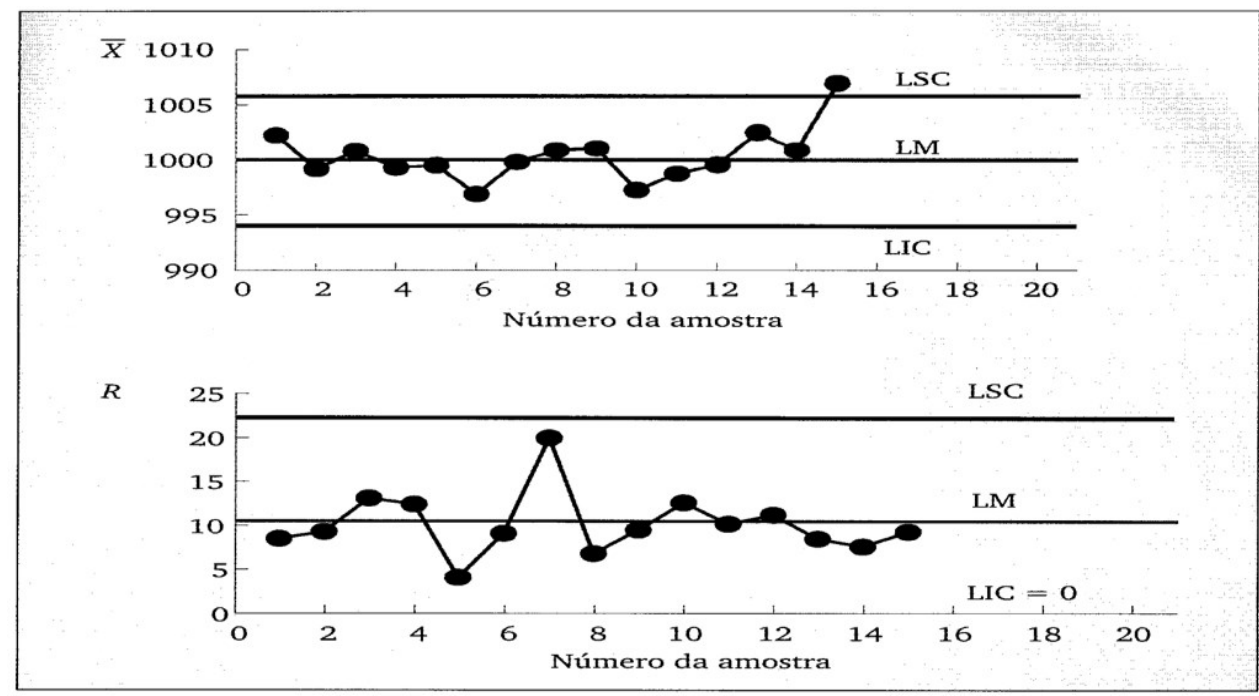

Fonte: Costa, Epprecht e Carpinetti (2005)

Sendo assim, os gráficos de servem para monitorar as possíveis causas especiais dos processos. Já o Diagrama de Causa e Efeito, também chamado de diagarama de Ishikawa, é uma excelente ferramenta de fácil compreensão e aplicação, demonstrando que todo problema do processo é o resultado de causas, disposta em seis grupos - Máquina, Mão-de-obra, Matéria-prima, Medida, Método e Meio (MIGUEL, 2001). A Figura 3 ilustra o Diagrama de Causa e Efeito, desenvolvido por Kaoru Ishikawa (1915- 1989).

Figura 3 - Diagrama de Causa e Efeito

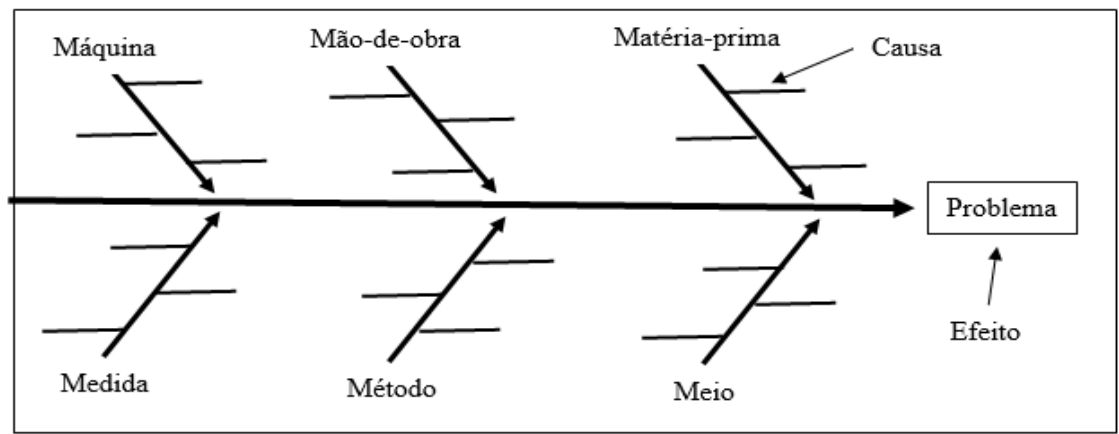

Fonte: Adaptado de Miguel (2001)

\section{A ESTATÍSTICA DAS CARTAS DE CONTROLE}

A melhoria contínua depende de vários fatores e métodos. Devido ao grande volume de produção e procedimentos, o método estatístico permite a análise e a interpretação dos dados de forma mais abrangente. Segundo Ramos (2000), os métodos estatísticos têm que ir além e associar-se a um pensamento sistêmico, que por sua vez possui alguns princípios norteadores, tais como:

a) Todo e qualquer trabalho executado é um processo, constituído de diversas etapas;

b) Todo processo está sujeito a variação, em maior ou menor quantidade, uma vez que isto é um fato da natureza; 
c) Sempre é possível melhorar um processo, mediante a eliminação da variação neste existente.

Montgomery (2009) afirma que há uma relação muito próxima entre os gráficos de controle e o teste de hipóteses. Se o valor atual da média se localiza entre os limites de controle, pode-se concluir que o processo está sob controle. Caso contrário, se a média excede os limites de controle o processo está fora de controle.

De certa maneira, o gráfico de controle é um teste de hipóteses do estado de controle estatístico, em que um ponto que se localiza entre os limites de controle equivale à não rejeição (aceitação) da hipótese de controle estatístico. E um ponto que se localiza fora dos limites de controle equivale à rejeição da hipótese de controle estatístico.

Assim sendo, a distribuição estatística que mais se ajusta a variação é a distribuição normal $\boldsymbol{Z} \sim \boldsymbol{N}(\boldsymbol{\mu}, \boldsymbol{\sigma})$. Portanto os limites de controle para o gráfico de $\overline{\mathbf{X}}$ possuem as denotações das Equações 4, 5 e 6 .

$$
\begin{aligned}
& L S C_{\bar{x}}=\mu_{\bar{x}}+3 \frac{\sigma_{x}}{\sqrt{n}} \\
& L M_{\bar{x}}=\mu_{\bar{x}} \\
& L I C_{\bar{x}}=\mu_{\bar{x}}-3 \frac{\sigma_{x}}{\sqrt{n}}
\end{aligned}
$$

(Equação 4)

(Equação 5)

(Equação 6)

Onde:

$\boldsymbol{\mu}_{\bar{x}}=$ média das médias amostrais;

$\sigma_{x}=$ desvio padrão não tendencioso;

$\boldsymbol{n}=$ número de elementos nos subgrupos amostrais.

Os limites de controle, com três desvios padrão de afastamento em relação à linha média, foram propostos por Shewhart, sendo também conhecido como "limite de 3 sigma" (COSTA, EPPRECHT, CARPINETTI, 2005; RIBEIRO, CATEN, 2012). Como o desvio padrão é tendencioso, comumente utiliza-se estimadores não tendenciosos $\left(\boldsymbol{\sigma}_{\overline{\boldsymbol{x}}}=\overline{\boldsymbol{S}} / \boldsymbol{c}_{\boldsymbol{4}}\right)$, onde $\overline{\boldsymbol{S}}$ é o desvio padrão das amostras, e $\boldsymbol{c}_{\mathbf{4}}$ o fator de correção). Ou seja, estimadores que são verdadeiros fatores de correção, que depende do tamanho $\mathrm{n}$ da amostra. Tais fatores de correção são tabelados, e variam conforme o número $\mathrm{n}$ de elementos na amostra, conforme Tabela 1.

Tabela 1 - Valores de $\mathrm{C}_{4}, \mathrm{~d}_{2}$ e $\mathrm{d}_{3}$

\begin{tabular}{cccccccc}
\hline $\mathbf{N}$ & $\mathbf{2}$ & $\mathbf{3}$ & $\mathbf{4}$ & $\mathbf{5}$ & $\mathbf{6}$ & $\mathbf{7}$ & $\mathbf{8}$ \\
\hline $\mathbf{c}_{4}$ & 0,798 & 0,886 & 0,921 & 0,94 & 0,952 & 0,959 & 0,965 \\
$\mathbf{d}_{\mathbf{2}}$ & 1,128 & 1,693 & 2,059 & 2,326 & 2,534 & 2,704 & 2,847 \\
$\mathbf{d}_{3}$ & 0,853 & 0,888 & 0,88 & 0,864 & 0,848 & 0,833 & 0,82 \\
\hline
\end{tabular}




\begin{tabular}{cccccccc}
\hline $\mathbf{N}$ & $\mathbf{9}$ & $\mathbf{1 0}$ & $\mathbf{1 1}$ & $\mathbf{1 2}$ & $\mathbf{1 3}$ & $\mathbf{1 4}$ & $\mathbf{1 5}$ \\
\hline $\mathbf{c}_{\mathbf{4}}$ & 0,969 & 0,973 & 0,975 & 0,978 & 0,979 & 0,981 & 0,982 \\
$\mathbf{d}_{\mathbf{2}}$ & 2,97 & 3,078 & 3,173 & 3,258 & 3,336 & 3,407 & 3,472 \\
$\mathbf{d}_{\mathbf{3}}$ & 0,808 & 0,797 & 0,787 & 0,778 & 0,77 & 0,763 & 0,756 \\
\hline
\end{tabular}

Fonte: Adaptado de Costa, Epprecht e Carpinetti (2005).

Já os fatores d2 e d3, apresentados na Tabela 1, são utilizados para calcular os limites de controle para o gráfico de $\mathrm{R}$, como estimadores corrigidos para $\boldsymbol{\mu}_{R}=$ $\boldsymbol{d}_{2} \boldsymbol{\sigma}$ e $\boldsymbol{\sigma}_{\boldsymbol{R}}=\boldsymbol{d}_{3} \boldsymbol{\sigma}$ e (para todo desvio padrão $\boldsymbol{\sigma}=\overline{\boldsymbol{R}} / \boldsymbol{d}_{2}$, sendo $\overline{\boldsymbol{R}}$ a média das médias da amplitude). Usualmente, encontram-se situados a três desvios padrão de afastamento da média, sendo assim, os limites são denotados pelas Equações 7,8 e 9.

$$
\begin{aligned}
& L S C_{R}=\mu_{R}+3 \sigma_{R} \\
& L M_{R}=\mu_{R} \\
& L I C_{R}=\mu_{R}-3 \sigma_{R}
\end{aligned}
$$

Onde:

$\boldsymbol{\mu}_{\boldsymbol{R}}=$ média das médias da amplitude;

$\boldsymbol{\sigma}_{\boldsymbol{R}}=$ desvio padrão não tendencioso;

Portanto, as Equações 7, 8 e 9 podem ser reescritas pelas Equações 10, 11 e 12 , respectivamente.

$$
\begin{aligned}
& L S C_{R}=d_{2} \sigma+3 d_{3} \sigma \\
& L M_{R}=d_{2} \sigma \\
& L I C_{R}=d_{2} \sigma-3 d_{3} \sigma
\end{aligned}
$$$$
\text { (Equação 10) }
$$$$
\text { (Equação 11) }
$$

Após definir estatisticamente os limites de controle, percebe-se que o gráfico da média é calculado em função da média e do desvio padrão. Diferentemente, o gráfico da amplitude depende apenas do desvio padrão.

Se o desvio padrão aumentar consideravelmente, as amplitudes amostrais tenderão a ser maiores. Quando isso acontecer e ultrapassar o limite superior de controle, apontará que o desvio padrão do processo aumentou e houve uma variabilidade no processo (COSTA, EPPRECHT, CARPINETTI, 2005; RIBEIRO, CATEN, 2012).

\section{ANÁLISE DE DESEMPENHO DOS GRÁFICOS}

Por meio da análise de desempenho dos gráficos, pode-se detectar perturbações no processo. Tais gráficos podem ser analisados isoladamente ou em conjunto, baseados em duas hipóteses mutuamente excludentes. Costa, Epprecht e Carpinetti (2005) denomina essas hipóteses em HO e H1. Se a hipótese HO é aceita 
como verdadeira todas as vezes que o valor de $\overline{\mathbf{X}}$ cair dentro dos limites de controle. Caso contrário, a hipótese $\mathrm{H} 1$ é aceita como verdadeira sempre que o valor de $\overline{\mathbf{X}}$ cair fora dos limites de controle.

Porventura, pode-se considerar, erroneamente, que o processo está em controle, sendo que está fora de controle (HO verdadeiro), e a representa o risco (probabilidade) deste evento. O gráfico indicará que houve um alarme falso, ou seja, um sinal indevido de que o processo está sob a influência de alguma causa especial. Se o erro consistir em um processo fora de controle (H1 verdadeiro), $\beta$ representa o risco, e não indica no gráfico. Teoricamente, tal erro é chamado de não-detecção (COSTA, EPPRECHT, CARPINETTI, 2005).

A Figura 4 esboça como seria a ocorrência de um alarme falso, descrito por meio dos testes de hipóteses. Matematicamente, o risco de cometer qualquer erro é dado pelas probabilidades expressas pelas Equações 13 e 14 .

$$
\begin{gathered}
\alpha=\operatorname{Pr}\left[\bar{X}>L S C_{\bar{X}} \text { ou } \bar{X}<L I C_{\bar{X}} \mid \mu=\mu_{\bar{x}}\right] \\
\beta=\operatorname{Pr}\left[L I C_{\bar{X}} \leq \bar{X} \leq L S C_{\bar{X}} \mid \mu \neq \mu_{\bar{x}}\right]
\end{gathered}
$$

Figura 4 - Gráfico de $\overline{\mathrm{X}}$ indicando um alarme falso

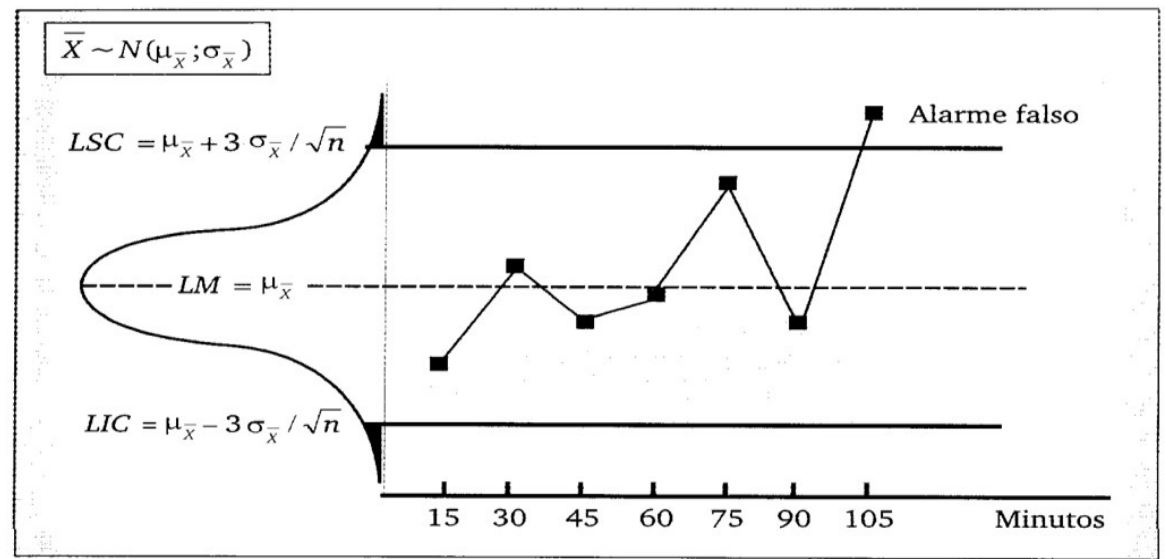

Fonte: Adaptado de Costa, Epprecht e Carpinetti (2005).

A análise de desempenho do gráfico de R é similar ao gráfico de . Contudo, a distribuição da probabilidade não é normal, mas sim assimétrica. Isso porque se adota a distribuição acumulada da amplitude relativa em função do limite inferior de controle não admitir valores negativos. Em termos formais, segundo Costa, Epprecht e Carpinetti (2005), admitem-se as probabilidades contidas nas Equações 15 e 16.

$$
\begin{aligned}
& \alpha=1-\operatorname{Pr}\left[L I C_{R} \leq R \leq L S C_{R} \mid \sigma=\sigma_{R}\right] \\
& \beta=\operatorname{Pr}\left[\operatorname{LIC}_{\mathrm{R}} \leq \mathrm{R} \leq \mathrm{LSC}_{\mathrm{R}} \mid \sigma \neq \sigma_{\mathrm{R}}\right]
\end{aligned}
$$




\section{METODOLOGIA}

Segundo Prodanov e Freitas (2013), a pesquisa científica é a realização de um estudo estruturado, sendo o método de abordagem do problema o que caracteriza o aspecto científico da investigação. A pesquisa sempre parte de um problema, no qual são levantadas hipóteses que podem ser confirmadas ou refutadas. Além disso, a investigação surge da necessidade de resolver problemas práticos e empíricos podendo levá-los à descoberta de princípios científicos.

No presente trabalho será implementada uma pesquisa quali-quanti. Qualitativa por desenvolver o diagrama de Ishikawa para avaliar as condições do processo de fabricação da microempresa e sua adequação aos requisitos do Inmetro adotados. Quantitativa, devido à aplicação dos métodos de controle estatístico, tais como a mesuração dos limites de controle, a curva de poder e a capacidade de processo.

Segundo Godoy (1995), o método quantitativo requer a definição clara das hipóteses de pesquisa, variáveis de estudo e quantificação de resultados. Enquanto o estudo qualitativo não requer a mensuração de eventos estudados, mas sim a avaliação das questões e perspectivas de pesquisa.

Nesse contexto, o estudo de caso é recomendável, uma vez que se investiga como e o porquê de os eventos ocorrerem em uma dada situação (YIN, 2005). Adicionalmente, Gil (2009) diz é aplicável em fenômenos específicos, onde se busca a descrição das situações em estudo e suas determinantes.

Tal pesquisa é baseada no método estatístico, possibilitando a formulação de hipóteses e a comprovação ou não das mesmas. Conforme Gil (2008), este método se fundamenta na aplicação da teoria estatística de probabilidade. Com base na utilização de testes estatísticos, é possível determinar, em termos numéricos, a probabilidade de acerto de determinada conclusão, bem como a margem de erro de um valor obtido. Para isso, foram usados os softwares Minitab ${ }^{\circledR}$ e Excel Microsoft ${ }^{\circledast}$ para a construção dos gráficos e limites de controle.

Neste sentido, os procedimentos estatísticos foram realizados em uma padaria, com o objetivo de identificar a variabilidade do processo produtivo por meio das cartas de controle estatísticos. O processo de pesquisa deu-se por meio da coleta de dados, tradução e interpretação dos mesmos, e por fim, com o auxílio das bases científicas teóricas, pode-se chegar a uma conclusão sobre os procedimentos adotados na padaria.

\section{CARACTERIZAÇÃO DA ÁREA DE ESTUDO}

Diante do contexto histórico e o atual, o presente estudo buscou evidenciar qual é a atual situação dos pães quanto à conformidade e padronização dos processos. Para isso é importante também descrever suas etapas. A Figura 5 expõe os cinco componentes da fabricação, destacando as principais atividades durante cada etapa na produção de pães. 
Figura 5 - Processo de fabricação de pães

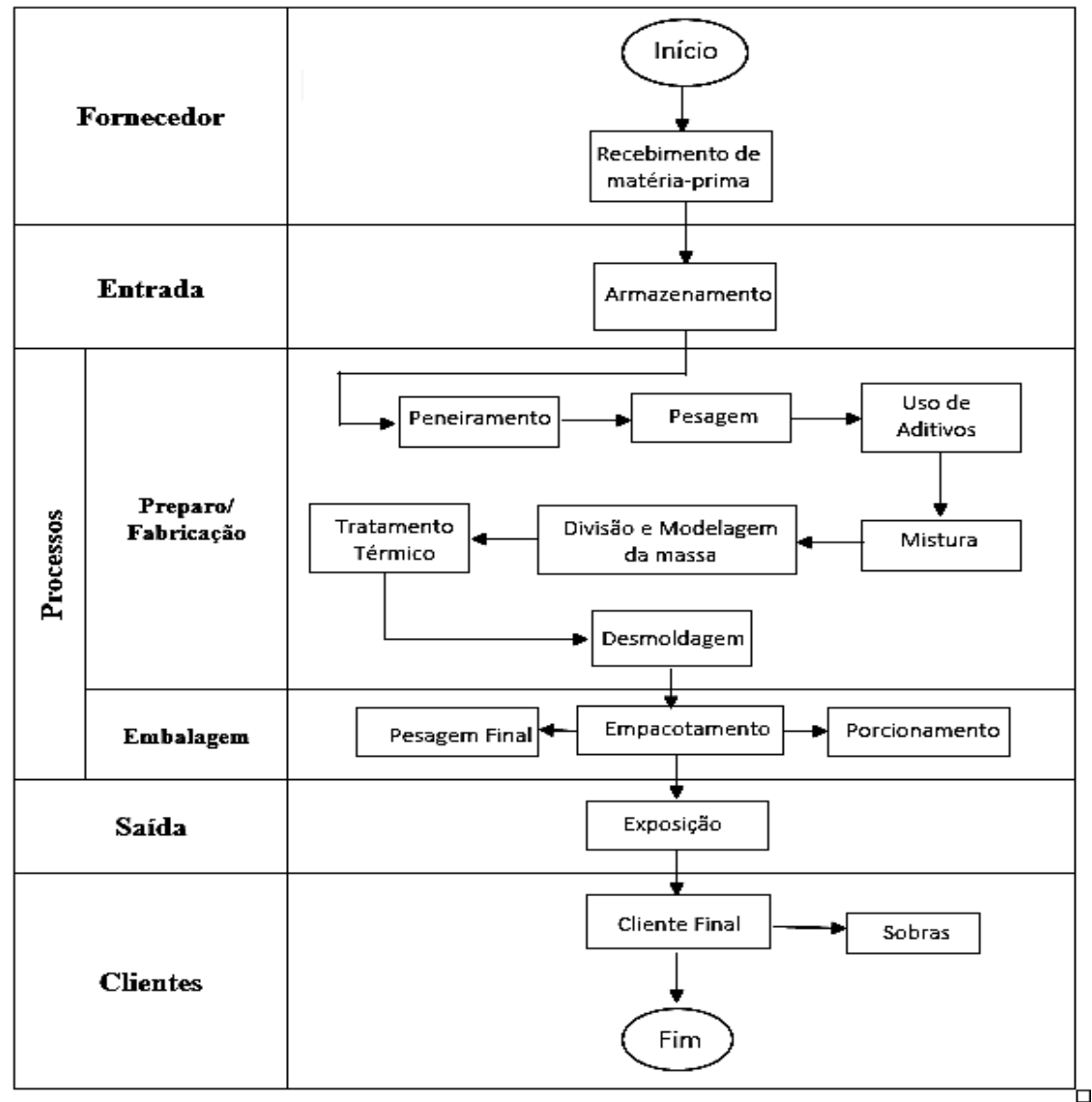

Fonte: Elaborado pelos autores (2017)

O mapeamento do processo torna-se fundamental para o levantamento das possíveis causas especiais que atuam no processo, e no peso resultante. Diante disso, quanto maior o número de atividades no processo, maior é sua vulnerabilidade a causas especiais.

Por meio das cartas de controle de Shewhart, pretende-se identificar a variabilidade do processo de fabricação dos pães, estabelecendo os limites de controle para o gráfico da média e para o gráfico da amplitude. Caso algum ponto caia fora dos limites de controle, por meio diagrama de causa e efeito, espera-se levantar as possíveis causa para tal efeito.

Tendo em vista que as cartas de controle levam em conta resultados estatísticos, o estudo também definiu o processo de acordo sua capacidade. Buscase identificar se o processo é incapaz, razoavelmente capaz ou capaz, de acordo com os índices de capacidade do processo.

\section{COLETA E MODELAGEM DOS DADOS}

Os dados foram coletados em uma microempresa de panificação no município de Congonhas - MG. Foram coletadas, ao longo de 6 dias, 25 amostras de forma consecutiva. Ou seja, a medida que se fabricava os pães (a cada nova fornada produzida). 
Com isso, garante-se que a coleta se dê em situações similares entre todas as fornadas analisadas. Parte-se, ainda, do princípio de que a produção é realizada com padronização. $\mathrm{O}$ tamanho das amostras está em conformidade com a portaria no 003/97 do Inmetro. Também leva em consideração o tamanho do lote.

Sendo assim, os lotes de análise consistem em tamanhos de 5 a 25 pães - que representam o número mínimo e máximo de produção por forma. Os valores de cada amostra (em gramas), constituída de 5 itens $(n=5)$, estão descritos na Tabela 2 com as respectivas médias e amplitudes calculadas.

Tabela 2 - Dados coletados

\begin{tabular}{|c|c|c|c|c|c|c|c|}
\hline $\mathrm{N}^{\circ}$ de Amostras (i) & $\mathrm{X}_{\mathrm{i} 1}$ & $\mathrm{X}_{\mathrm{i} 2}$ & $\mathrm{X}_{\mathrm{i3}}$ & $\mathrm{X}_{\mathrm{i} 4}$ & $\mathrm{X}_{\mathrm{is}}$ & $\overline{\mathrm{X}}$ & $\mathrm{R}$ \\
\hline 1 & 46 & 48 & 48 & 46 & 48 & 47,2 & 2,00 \\
\hline 2 & 50 & 50 & 50 & 48 & 52 & 50 & 4,00 \\
\hline 3 & 52 & 48 & 50 & 50 & 50 & 50 & 4,00 \\
\hline 4 & 44 & 46 & 44 & 46 & 44 & 44,8 & 2,00 \\
\hline 5 & 50 & 48 & 50 & 52 & 50 & 50 & 4,00 \\
\hline 6 & 50 & 50 & 48 & 50 & 48 & 49,2 & 2,00 \\
\hline 7 & 52 & 52 & 48 & 54 & 48 & 50,8 & 6,00 \\
\hline 8 & 42 & 50 & 50 & 50 & 46 & 47,6 & 8,00 \\
\hline 9 & 50 & 50 & 50 & 54 & 48 & 50,4 & 6,00 \\
\hline 10 & 50 & 38 & 46 & 46 & 48 & 45,6 & 12,00 \\
\hline 11 & 50 & 52 & 48 & 48 & 44 & 48,4 & 8,00 \\
\hline 12 & 48 & 48 & 50 & 48 & 50 & 48,8 & 2,00 \\
\hline 13 & 48 & 48 & 48 & 50 & 46 & 48 & 4,00 \\
\hline 14 & 50 & 48 & 46 & 48 & 46 & 47,6 & 4,00 \\
\hline 15 & 44 & 46 & 46 & 40 & 48 & 44,8 & 8,00 \\
\hline 16 & 52 & 48 & 48 & 48 & 48 & 48,8 & 4,00 \\
\hline 17 & 48 & 46 & 44 & 46 & 50 & 46,8 & 6,00 \\
\hline 18 & 48 & 50 & 46 & 48 & 46 & 47,6 & 4,00 \\
\hline 19 & 44 & 46 & 46 & 42 & 40 & 43,6 & 6,00 \\
\hline 20 & 48 & 48 & 48 & 48 & 40 & 46,4 & 8,00 \\
\hline 21 & 48 & 46 & 48 & 48 & 48 & 47,6 & 2,00 \\
\hline 22 & 50 & 52 & 48 & 48 & 46 & 48,8 & 6,00 \\
\hline 23 & 48 & 46 & 46 & 44 & 44 & 45,6 & 4,00 \\
\hline 24 & 44 & 46 & 46 & 42 & 44 & 44,4 & 4,00 \\
\hline 25 & 40 & 44 & 44 & 44 & 40 & 42,4 & 4,00 \\
\hline \multicolumn{6}{|c|}{ Média das Médias } & 47,408 & 4,96 \\
\hline
\end{tabular}

Fonte: Pesquisa de campo (2017).

\section{APRESENTAÇÃO E ANÁLISE DE RESULTADOS}

Após a coleta e modelamento dos dados, os mesmos foram aplicados no Minitab ${ }^{\circledast}$ e Action (suplemento para o Excel Microsoft ${ }^{\circledR}$ ) para elaboração das cartas de controle. Inicialmente, buscou-se verificar o poder de detecção em amostras compostas pela pesagem de cinco pães $(n=5)$ através da Figura 6 . 
Figura 6 - Curva de poder (amostra $n=5)$

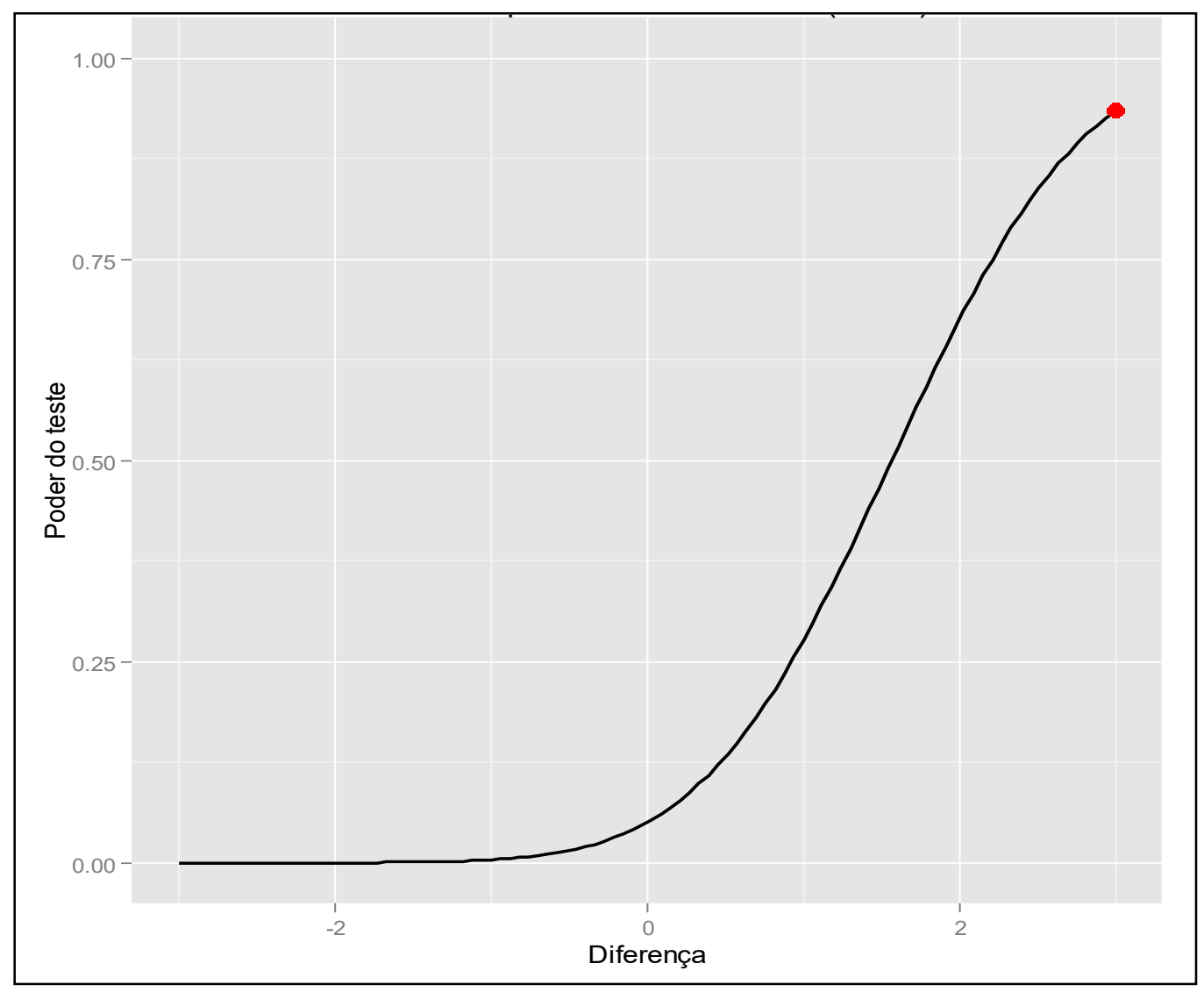

Fonte: Elaborado pelos autores com base no suplemento Action do software Microsoft Excel (2017).

Pode-se concluir que o poder de detecção é de aproximadamente 0,93 (93\%) para as amostras $n=5$, o que demonstra a capacidade do experimento em representar o processo em estudo. Tal detecção foi realizada caso a média tivesse um deslocamento de até 3 pontos para cima, com nível de confiança em 0,95 (95\%).

Além disso, foi desconsiderado qualquer deslocamento para baixo do valor da média amostra, uma vez que média amostral está abaixo do que é especificado (50g). O deslocamento abaixo da média só agravaria a situação, deixando mais pontos fora dos limites de controle.

Posteriormente, adotando-se 3 desvios padrão da média $(3 \sigma)$. Assim, obtevese que o limite superior de controle foi igual a 50,269 gramas e o limite inferior de controle foi 44,547 gramas, considerando a média das médias igual a 47,408 gramas, como mostra a Figura 7. 
Figura 7 - Cartas de controle $\overline{\mathbf{X}}$ e R

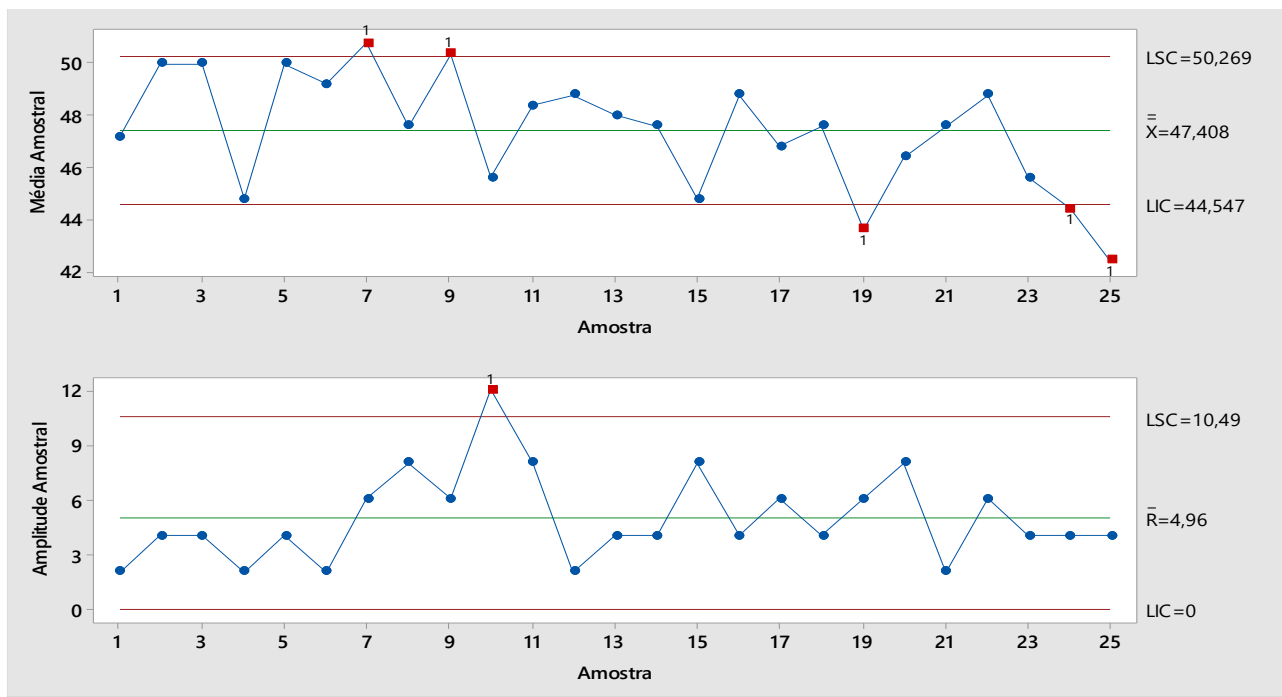

Fonte: Elaborado pelos autores à partir do software Minitab (2017)

Ao analisar a carta de controle das médias e a carta de controle da amplitude, constata-se que o processo está fora de controle. No que se refere a carta de controle de $\overline{\mathbf{X}}, 5$ das 25 amostras coletadas mostraram-se além do limites de controle. Dentre as amostras fora dos limites de controle estão as 7, 9, 19, 24, 25.

Para a carta de controle de $\mathrm{R}$, apenas a amostra 10 excedeu o limite superior de controle igual a 10,49. O valor médio das amplitudes foi igual a 4,96, e o limite inferior de controle, como foi negativo, teve o valor de zero adotado.

O processo só estaria estável caso fossem eliminadas 7 amostras (amostra 10, $25,19,24,15,4$, e 23 , respectivamente). Tendo em vista que foram coletadas apenas 25 amostras, eliminar 7 amostras poderia invalidar o procedimento, devido ao número insuficiente de amostras para uma conclusão. Uma alternativa seria eliminar as causas especiais, e posteriormente coletar novas amostras para fins de averiguação.

Dando continuidade, buscou-se a avaliação da capacidade do processo. Para isso, foi gerado o relatório de capacidade do processo através do Minitab, como demonstrado na Figura 8. 
Figura 8 - Relatório da capacidade do processo

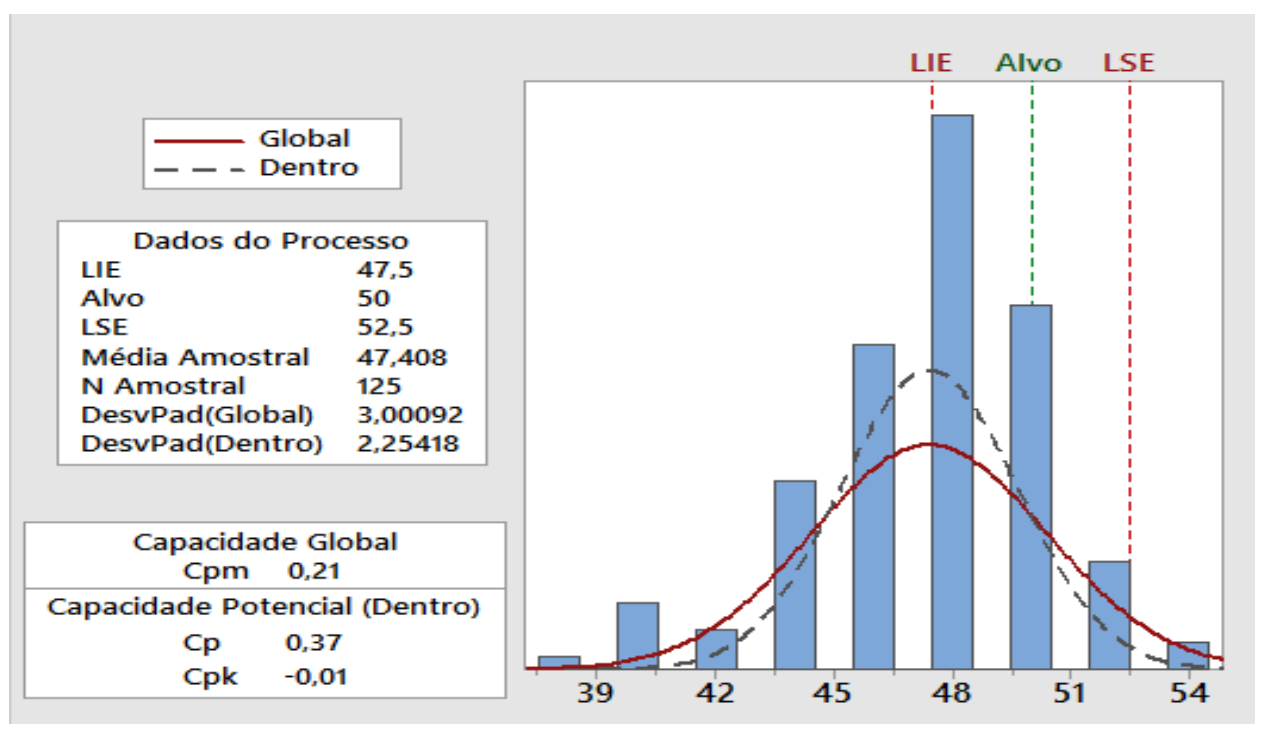

Fonte: Elaborado pelos autores a partir do software Minitab (2017)

Assim, quanto ao nível de capacidade, o processo mostrou ser incapaz uma vez que o índice Cpk foi de -0,01 (negativo) e ser menor do que 1. Tal fato deve-se a média das amostras ser inferior ao limite inferior de especificação, que tem como referência 47,5g. Este valor para o limite inferior de especificação e também para o limite superior tem como base a portaria no 003/97 do Inmetro, que determina que a tolerância do peso seja de $5 \%$ para mais ou menos. Sendo assim, os limites inferior e superior de especificação são, respectivamente, de $47,5 \mathrm{~g}$ e 52,5g, tendo como valor alvo $50 \mathrm{~g}$.

Adicionalmente, o processo também se mostrou incapaz pelos índices $C p=0,37$ e $C p m=0,21$, que ficaram bem baixos (menores que 1). A Figura 8 ilustra todos os índices descritos, bem como a distribuição normal do processo. Pela distribuição normal, nota-se um grande deslocamento da média do processo se comparado com o valor alvo.

Percebe-se também na Figura 8, as curvas de probabilidade dos itens. A curva "global" considera todos os subgrupos de forma global, propriamente dito; já a curva "dentro" contempla o desvio padrão dentro de cada subgrupo. Como a distribuição normal tem como parâmetros a média e o desvio, é nítida a distância entre a média do processo e o valor alvo. Sendo que o limite inferior de especificação fica bem próximo a média, confirmando assim a incapacidade do processo quanto ao controle estatístico.

Em decorrência das amostras fora de controle, principalmente a amostra 10 que teve a amplitude igual a 12, foi elaborado um diagrama de causa e efeito afim de identificar as possíveis causas para tal descontrole do processo. A Figura 9 apresenta o diagrama de Ishikawa, entre os quais foram levantadas, através de brainstorming com a equipe de produção, as três principais causas de cada um dos seis segmentos (método, matéria-prima, máquina, meio, medida, mão-de-obra). 
Figura 9 - Diagrama de causa e efeito

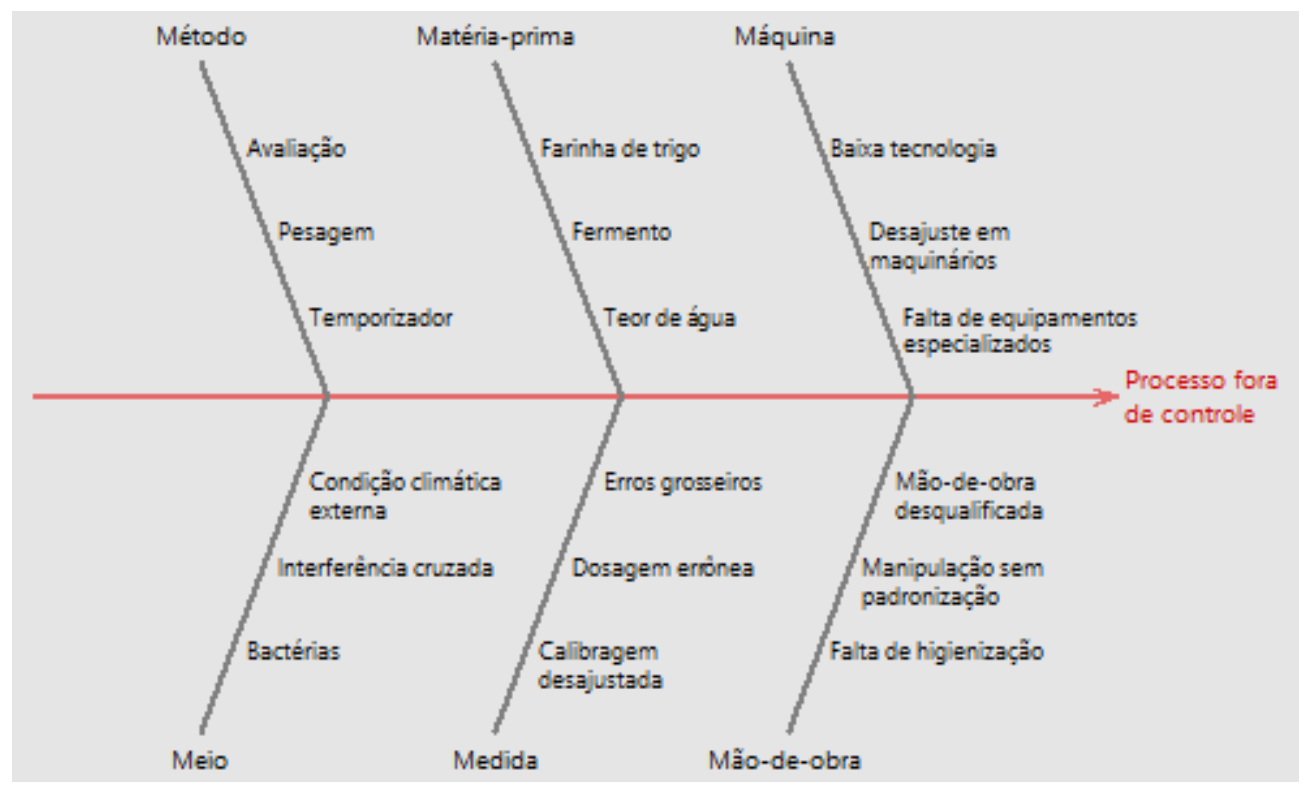

Fonte: Elaborado pelos autores à partir do software Minitab (2017)

Basicamente, as variantes de processo estão atreladas à necessidade de padronização de produção. É importante assegurar que as condições produtivas não sofram variações, e sigam procedimentos operacionais previamente testados e validados para a fabricação de pães. Tais não-conformidades repercutem na variabilidade de peso nos pães comercializados. Isso indica que a venda de pães seja feita somente pelo peso, conforme estabelece o Inmetro, de modo que haja uma relação justa e satisfatória com o preço cobrado dos clientes.

\section{CONSIDERAÇÕES FINAIS}

O presente estudo teve como finalidade avaliar a produção de pães de uma pequena padaria localizada em Congonhas-MG. Para isso, utilizou-se da metodologia de Controle Estatístico do Processo (CEP), procedendo-se análise de poder do experimento amostral, além de elaborar cartas de controle - tanto para média, quanto para amplitude [e analisar a capacidade de processo pelos índices Cpk, Cp e Cpm.

Diante de todo estudo realizado, foi possível concluir que o processo não se encontra sob controle. Consequentemente, foram identificadas as causas para a falta de controle através do diagrama de Ishikawa. Estas apontaram a falha na padronização de procedimentos operacionais de fabricação.

A realidade confirmou a importância de se vender os pães por peso, como forma de precificar o produto de maneira mais justa junto aos clientes, o que lhes gera satisfação. Assim, é obrigatório seguir as recomendações do Inmetro para a comercialização por peso, e não por unidade, como era feito antes de aprovada a portaria no 003/1997.

A garantia de produção conforme requer o desenvolvimento de procedimento operacional padrão. Para isso, é necessário garantir condições 
ambientais uniformes de fabricação, além de compra de matéria-prima de fornecedores validados e treinamento e monitoramento de funcionários. Isso requer esforço financeiro, além da quebra de hábitos culturais. Ou seja, implementar tais mudanças pode remeter a um dispêndio importante por parte da empresa, o que pode gerar um obstáculo à mudança.

O estudo comprova a validade da metodologia do CEP para análise de processos e tomada de decisão. Pode-se apontar como possibilidade de estudo a viabilidade em se implementar os Procedimentos Operacionais Padrão (POPs). Para essa análise, critérios financeiros e organizacionais certamente são necessários, uma vez que requer o investimento e implica, no futuro, no uso de procedimentos padronizados indepedente de quem o faça, o que também é uma condição importante à empresa. 


\title{
Analysis of variability in bread production in a small bakery with the assistance of statistical quality control
}

\author{
ABSTRACT
}

In production processes the standardization of quality is essential, both for greater efficiency and efficiency of the organization, and for customer satisfaction. This article aims to apply quality tools in a small bakery in the city of Congonhas - MG. Specifically, it seeks to identify the variability of weight in the manufacture of French bread and the possible special causes present in the process. Even with the adoption of the Inmetro standard, such study proposes to define the best way to market bread: per unit or by weight. For this, the case study was done through methods of statistical quality control, where the reality was evaluated by Ishikawa diagram, control charts, power curve and process capability. As a result, the statistical control mechanisms of the process indicated a lack of control in the manufacturing procedure, which indicates the need to sell by weight.

KEYWORDS: Statistical quality control. Quality tools. Variability. Special causes. Inmetro. 


\section{REFERÊNCIAS}

CARNEIRO NETO, W. Controle estatístico de processo CEP. Recife: UPE-POLI; 2003.

CARVALHO, M. M.; PALADIN, E.P. Gestão da qualidade: teoria e casos. Rio de Janeiro: Editora Campus, 2005. 355p.

CORTIVO, Z. D. Aplicação do Controle Estatístico de Processo em seqüências curtas de produção e análise estatística de processo através do planejamento econômico. [Dissertação] Curitiba: Universidade Federal do Paraná; 2005.

COSTA, A. F. B.; EPPRECHT, K.; CARPINETTI, L. C. R. Controle estatístico de qualidade. 2a. ed. São Paulo: Atlas, 2005. 333 p.

CHRISTINO E. M.; BONDUELLE G. M.; IWAKIRI, S. Aplicação de Cartas de Controle no Processo de Fabricação de Pisos Sólidos de Tauari (Cauratari oblongifolia). Cerne, Lavras, v. 16, n. 3, jul./set. 2010. p. 299-304.

DINIZ, M. G. Desmistificando o controle estatístico de processo. São Paulo: Artliber; 2001.

GIL, A. C. Métodos e técnicas de pesquisa social. 6. ed. São Paulo: Atlas, 2008. $196 \mathrm{p}$.

GIL, A. C. Como elaborar projetos de pesquisa. 4. ed. São Paulo: Atlas, 2009. 175 p.

GODOY, A. S. Introdução à pesquisa qualitativa e suas possibilidades. Revista de Administração de Empresas da EAESP/FGV, São Paulo, v. 35, n. 2, mar./abr. 1995. p. 57-63.

INMETRO. Portaria no 017, de 25 de janeiro de 1994. Rio de Janeiro: INMETRO, 1994. 1 p. Disponível em:

<http://www.inmetro.gov.br/legislacao/rtac/pdf/RTAC000167.pdf>. Acesso em: 25 dezembro 2016.

INMETRO. Portaria no 003, de 10 de janeiro de 1997. Rio de Janeiro: INMETRO, 1997. 1 p. Disponível em:

<http://www.inmetro.gov.br/legislacao/rtac/pdf/RTAC000219.pdf>. Acesso em: 25 dezembro 2016. 
INMETRO. Pão francês será vendido por peso. Rio de Janeiro: INMETRO, 2006. Disponível em: <http://www.inmetro.gov.br/imprensa/releases/paoPeso.asp>. Acesso em: 25 dezembro 2016.

INMETRO. Portaria no 63, de 15 de março de 2006. Rio de Janeiro: INMETRO, 2006a. 2 p. Disponível em: <http://www.inmetro.gov.br/rtac/pdf/RTAC001010.pdf>. Acesso em: 25 dezembro 2016.

INMETRO. Portaria no 146, de 20 de junho de 2006. Rio de Janeiro: INMETRO, 2006b. 1 p. Disponível em:

<http://www.inmetro.gov.br/legislacao/rtac/pdf/RTAC001032.pdf>. Acesso em: 25 dezembro 2016.

JURAN, J. M. A qualidade desde o projeto. São Paulo: Pioneira, 1992.

LIMA, A.A.N.; LIMA, J.R.; SILVA, J.L.; ALENCAR, J.R.B.; SOARES-SOBRINHO, J.L.; LIMA, L.G.; ROLIM-NETO, P.J. Aplicação do controle estatístico de processo na indústria farmacêutica. Rev. Ciênc. Farm. Básica Apl., v. 27, n.3, 2006. p.177-187.

LINDINO, C. A.; NUNES, O. L. G. Implantação de controle estatístico em determinação de nitrogênio total e proteína bruta em peito de frango. Acta Scientiarum. Technology, v. 33, n. 1, 2011. crossref

MIGUEL, P. A. C. Qualidade: enfoques e ferramentas. 1ạ. ed. São Paulo: Artliber, 2001. 272 p.

MONTGOMERY, D. C. Introdução ao controle estatístico da qualidade. 4.ed. Rio de Janeiro: LTC, 2009. 513 p.

MOREIRA, D. A. Administração da produção e operações. São Paulo: Pioneira; 2004. p.561.

NORONHA, R. H. F.; SILVA, R. P.; CHIODEROLI, C. A.; SANTOS, E. P.; CASSIA, M. T. Controle estatístico aplicado ao processo de colheita mecanizada diurna e noturna de cana-de-açúcar. Bragantia, Campinas, v. 70, n. 4, 2011. p.931-938. crossref

PALADINI, E. P. Avaliação estratégica da qualidade. São Paulo: Atlas; 2002. p.64114. 
PARANTHAMAN, D. Controle de qualidade. São Paulo: McGrawHill Ltda; 1990. p.118-212.

PRODANOV, C. C.; FREITAS, E. C. D. Metodologia do trabalho científico: métodos e técnicas da pesquisa e do trabalho científico. 2a. ed. Novo Hamburgo: Feevale, 2013. $271 \mathrm{p}$.

RAMOS, A. W. CEP para processos contínuos e em bateladas. 1‥ ed. São Paulo: Edgard Blucher, 2000. 130 p.

RIBEIRO, J. L. D.; CATEN, C. S. Controle Estatístico do Processo. Série Monografia Qualidade. FEENG/UFRGS - Fundação Empresa Escola de Engenharia da UFRGSUniversidade Federal do Rio Grande do Sul. Escola de Engenharia. Programa de Pós-Graduação em Engenharia de Produção. Porto Alegre, RS.2012.

SANTOS, J. K. C.; BATISTA, N. S. Controle estatístico de processo: uma ferramenta para validação do processo de envase. [Trabalho de Conclusão de Curso]. Recife: Universidade Federal de Pernambuco. LAFEPE - Laboratório Farmacêutico do Estado de Pernambuco; 2005

SEBRAE. Boas práticas na panificação e na confeitaria - da produção ao ponto de venda. 1a. ed. Brasília: SEBRAE, 2010. 102 p. Disponível em:

<http://www.abip.org.br/site/wp-

content/uploads/2016/01/cartilhafinalizada.pdf>. Acesso em: 25 dezembro 2016.

SILVA, R. P. et al. Variabilidade espacial e controle estatístico do processo de perdas na colheita mecanizada do algodoeiro. Engenharia Agrícola. Associação Brasileira de Engenharia Agrícola, v. 27, n. 3, p. 742-752, 2007. crossref

THOMPSON JR.; KORONACKI, J. Statistical process control for quality improvement. London: Chapman \& Hall; 1993.

VIEIRA, S. Estatística para a qualidade: como avaliar com precisão a qualidade em produtos e serviços. Rio de Janeiro: Elsevier; 1999.

YIN, R. K. Estudo de caso: planejamento e métodos. 3. ed. Porto Alegre: Bookman, 2005. 212 p. 
Recebido: 14 maio. 2017

Aprovado: 19 dez. 2017

DOI: 10.3895/gi.v13n4.5905

Como citar:

SOUSA JUNIOR, E. A.; LONGHINI, T. M.; COSTA, H. I. S. C. Análise da variabilidade na produção de pães em uma pequena padaria com o auxílio do controle estatístico da qualidade. R. Gest. Industr., Ponta

Grossa, v. 13, n. 4, p. 223-245, out./dez. 2017. Disponível em: <https://periodicos.utfpr.edu.br/rgi >. Acesso em: XXX.

Correspondência:

Edvan Araújo de Sousa Júnio

Rua Casa de Pedra n 378 Casa A, Vila Andreza, Congonhas, Minas Gerais, Brasil.

Direito autoral: Este artigo está licenciado sob os termos da Licença Creative Commons-Atribuição 4.0

Internacional.

(c) (1) 